\title{
PARIKH TEST SETS FOR COMMUTATIVE LANGUAGES
}

\author{
ŠTĚPÁN HOLUB
}

\begin{abstract}
A set $T \subseteq L$ is a Parikh test set of $L$ iff $c(T)$ is a test set of $c(L)$. We give an effective characterization of Parikh test sets for arbitrary
\end{abstract} commutative language.

\section{INTRODUCTION}

Commutative languages represent a class of languages with the rare property that an upper bound for the cardinality of their test sets is known (see [2], [1]). Namely, each commutative language has a test set with cardinality at most $3 n^{2}$, where $n$ is the number of letters. Moreover, the bound is optimal up to a constant, since for each $n$ there is a language the smallest test set of which has cardinality $\frac{1}{9} n^{2}$.

On the other hand, there is a large class of commutative languages, which have a linear test set. The motivation of this paper is to try to describe, which commutative languages have only test sets of quadratic size.

Since a commutative language is fully described by the set of its Parikh vectors, it is quite natural to investigate the size of test sets in terms of the cardinality of its Parikh image, i.e. the number of different Parikh vectors present in the language. We shall call this number the size of a Parikh test set of the language. It turns out that even for the Parikh size, the lower bound is $\Omega\left(n^{2}\right)$.

Parikh basis of a language and the information about common occurence of letters in a word is natural finite information about a language over finite alphabet. In this paper we show that it is sufficient for an effective characterization of Parikh test set for arbitrary commutative language.

\section{Definitions AND KNOWN FACTS}

Commutative closure of a word $u=\ell_{1} \ldots \ell_{n}$ is the language

$$
c(u)=\left\{\ell_{\sigma(1)} \ldots \ell_{\sigma(n)} \mid \sigma \in S_{n}\right\} .
$$

Commutative closure of a language is

$$
c(L)=\bigcup_{u \in L} c(u)
$$

Language $L$ is said to be commutative iff $L=c(L)$. Two morphisms agree on a language $L$ iff $g(u)=h(u)$ for any $u \in L$, write $g \equiv_{L} h$. A subset $T$ of the language $L$ is called its test set iff for any two morphisms $g, h$

$$
g \equiv_{L} h \quad \Leftrightarrow \quad g \equiv_{T} h .
$$

The set of letters occurring in a word $w$ is denoted by $\operatorname{alph}(w)$. 
Parikh vector of a word $u=a_{1}^{k_{1}} \ldots a_{n}^{k_{n}}$ is defined by

$$
\Psi(u)=\left(k_{1}, \ldots, k_{n}\right) .
$$

Let $X$ be a given alphabet, $|X|=n$. Then a commutative language $c(L)$ is given uniquely by the set of $\Psi[L] \subseteq \mathbb{N}^{n}$ of its Parikh vectors.

A basis of a commutative language $L$ is a set $B \subseteq L$ such that $\Psi[B]$ is a basis of the vector space over $\mathbb{Q}$ generated by $\Psi[L]$.

Two morphisms $g$ and $h$ are said to agree lengthwise on $L$ iff $|g(u)|=|h(u)|$ for all $u \in L$. $L$.

Note that morphisms $g$ and $h$ agree lengthwise on $L$ iff they agree on a basis of

The following results have been obtained for commutative languages so far.

Theorem 1 (see [1]). Any commutative language over $n$ letters has a test set of cardinality at most $3 n^{2}$.

There is a commutative language over $3 n$ letters, the smallest test set of which has cardinality at least $n^{2}$.

Theorem 2 (see [2]). The commutative language $c\left(a_{1}, \ldots, a_{n}\right)$ has a test set of cardinality at most 5 n.

The commutative language $c\left(a_{1}^{k_{1}} \cdots a_{n}^{k_{n}}\right)$ has a test set of cardinality at most $11 n$.

Each test set of the commutative language $c\left(a_{1} \cdots a_{n}\right)$ has cardinality at least $n-1$.

\section{PARIKH TEST SETS}

In this section we accomplish the main task of this paper. Given a commutative language we shall decide what is the smallest Parikh size of its test set.

Since a commutative language is fully described by its Parikh image, it is quite natural to measure the size of its test set by its Parikh size. We therefore introduce the following notion. A set $T \subseteq L$ is a Parikh test set of $L$ iff $c(T)$ is a test set of $c(L)$.

It is straightforward to see that the relationship between the size of Parikh test set and classical test set obeys two very broad bounds (the second one follows from Theorem 2)

- Parikh test sets give a lower bound to cardinality of test sets

- If $T$ is a Parikh test set of $L$, then $L$ has a test set of cardinality

$$
11 \sum_{t \in T}|\operatorname{alph}(t)| \text {. }
$$

The basic description of a language $L$ over an alphabet $X$, by which we shall describe the Parikh test sets, is an undirected graph called coincidence graph, and denoted $G(L)$. It is defined as follows. The set of vertices of the graph is the alphabet $X$. The set of edges $E=E(L)$ is defined by $(a, b) \in E$ if and only if there is a word $w$ in $L$ such that $a b$ is a factor of $c(w)$. The graph admits loops, since we do not require $a \neq b$. Therefore the loop $(a, a)$ is in $E$ if and only if $L$ contains a word with at least two occurrences of the letter $a$.

The sequence of vertices $x_{0}, \ldots, x_{m}$ is called a path if $\left(x_{0}, x_{1}\right), \ldots,\left(x_{m-1}, x_{m}\right) \in$ $E$. The length of such a path is $m$, i.e., the number of edges.

We are going to show that Parikh test sets can be fully caracterised by coincidence graphs: 
Theorem 3. If $G(L), G(T)$, and a basis of $L$ are effectively given, then it is decidable whether $T$ is a Parikh test set of $L$.

First we need some more definitions. Let $L$ be a language over $X=\left\{a_{1}, \ldots, a_{n}\right\}$. Given morphisms $g, h: X \rightarrow A^{*}$, define

$$
D(g, h)=\left\{a_{i}|| g\left(a_{i}\right)|\neq| h\left(a_{i}\right) \mid\right\} .
$$

A subset $D \subseteq X$ is called a difference support of $L$ iff there are morphisms $g, h$ which are length equivalent on $L$, and $D=D(g, h)$.

Difference supports have the following equivalent characterization, which allows to find all of them by means of linear algebra.

Lemma 1. Let $L$ be a language over $X=\left\{a_{1}, \ldots, a_{n}\right\}$ and $B$ a basis of $L . D \subseteq X$ is a difference support of $L$ if and only if there is a vector $\left(d_{1}, \ldots, d_{n}\right) \in \mathbb{Q}^{n}$, such that

$$
D=\left\{a_{i} \mid d_{i} \neq 0\right\}
$$

and $b \cdot d=0$ for each $b \in \Psi[B]$.

Proof. If $D=D(g, h)$ for length equivalent morphisms, put $d_{i}=\left|g\left(a_{i}\right)\right|-\left|h\left(a_{i}\right)\right|$.

Let on the other hand $D$ be the support of a vector $d$, which solves the system of equations

$$
b \cdot d=0, \quad \text { for all } b \in \Psi[B] .
$$

We can choose $d$ such that $d \in \mathbb{Z}^{n}$. Then $D=D(g, h)$ for any $g, h$ defined by

$$
g\left(a_{i}\right)=a^{k_{i}}, \quad h\left(a_{i}\right)=a^{\ell_{i}}
$$

with $k_{i}-\ell_{i}=d_{i}$ for all $i=1, \ldots, n$. It is not difficult ot see that $g \equiv_{L} h$.

Given a set $D \subseteq X$ we say that $x_{0}, \ldots, x_{k}$ is a $D$-path if for each $0 \leq i \leq k-1$ at least one of vertices $x_{i}, x_{i+1}$ is in $D$ (i.e. there is no edge in the path with both vertices out of $D$ ). If there is a $D$-path between $a$ and $b$, we say that they are D-connected.

Consider now a language $L$ over $X$ and morphisms $g$ and $h$, which agree on $L$. We shall always suppose that $g(x) h(x)$ is nonempty for each $x \in X$.

The most obvious case in which two morphisms $g$ and $h$ agree on commutative closure of a word $w$ is when $g(a)=h(a)$ for all $a \in \operatorname{alph}(w)$.

The following lemmas describe two other possibilities. Proofs may be found for example in [2].

Lemma 2. Let $g$ and $h$ agree on $c(a b)$, and $g(a) \neq h(a)$. Suppose that $g(a)$ and $g(b)$ do not commute. Then there are unique nonempty words $r$, s, such that $r s$ is primitive, and

$$
\begin{array}{ll}
g(a)=(r s)^{i} r & g(b)=(s r)^{j} s \\
h(a)=(r s)^{i+k} r & h(b)=(s r)^{j-k} s
\end{array}
$$

for some integers $i, j, k$.

Lemma 3. Let $g$ and $h$ agree on $c(w)$, where $|w| \geq 3$, and $g(a) \neq h(a)$ for at least one $a \in \operatorname{alph}(w)$. Then all words in

$$
g[\operatorname{alph}(w)] \cup h[\operatorname{alph}(w)]
$$

commute. 
Fix now morphisms $g$ and $h$ which agree on a commutative language $L$ and put $D=D(g, h)$.

For each primitive $t$ define

$$
P_{t}=\left\{a \mid g(a), h(a) \in t^{*}\right\},
$$

and let

$$
P=\bigcup_{t} P_{t}
$$

Denote

$$
Z=X \backslash D=\{a \mid g(a)=h(a)\} .
$$

Define also a symmetric relation $\sim$ on $X$ by $a \sim b$ iff $a$ and $b$ satisfy conditions of Lemma 2. Denote

$$
S=\{a \mid a \sim b \text { for some } b\} .
$$

From the definitions, and from Lemma 2 and Lemma 3, we deduce the following claim.

Lemma 4. If $(a, b) \in E$ then at least on of the following conditions is satisfied.

- $a, b \in Z$; or

- $a, b \in P_{t}$ for some $t$; or

- $a \sim b$.

Moreover, $Z \subseteq P$, and $S$ and $P$ are disjoint.

Since the words $r$ and $s$ in Lemma 2 are given uniquely, we have also the following properties of $\sim$.

\section{Lemma 5.}

(1) If $x_{0} \sim x_{1} \sim \cdots \sim x_{k}$, then $x_{0} \sim x_{j}$ for $1 \leq j \leq k$ odd.

(2) If $x_{0} \sim x_{1} \sim \cdots \sim x_{k} \sim x_{0}$ then $k$ is odd.

We point out the following facts about paths.

Lemma 6. If $x_{0}, \ldots, x_{k}, x_{0}$ is a path in $G(L)$ of odd length, and $x_{0} \in D$ then $x_{0} \in P_{t}$ for some $t$.

Lemma 7. If $x_{0}, \ldots, x_{k}$ is a D-path in $G(L)$ then either $x_{i} \in S$ for all $i=0, \ldots, k$ or $x_{i} \in P_{t}$ for all $i=0, \ldots, k$ and some primitive $t$.

We can now formulate our criterion for Parikh test sets.

Theorem 4. $T \subseteq L$ is a Parikh test set of $L$ if and only if $T$ contains a basis of $L$, and for each difference support $D$ the graph $G(T)$ satisfies the following two conditions.

(A) If letters $a$ and $b$ are D-connected in $G(L)$ then they are D-connected in $G(T)$ as well.

(B) If for some $a \in D$ there is a path from a to a of odd length in $G(L)$ then there is such a path also in $G(T)$.

Proof.

1. We first prove that our criterion of the test set is sufficient. Let $g, h$ be two morphisms which agree on $c(T)$. We want to show that they agree on $c(w)$ for each $w \in L$. 
Pick a word $w \in L$. Since $T$ contains a basis of $L$, the morphisms agree lengthwise on $c(L)$. If $g(a)=h(a)$ for each $a \in \operatorname{alph}(w)$ then there is nothing to prove. Let therefore $d \in \operatorname{alph}(w)$ be a letter, for which $g(d) \neq h(d)$.

Consider a letter $a \in \operatorname{alph}(w)$. The letters $a$ and $d$ are $D$-connected in $G(L)$, therefore, by (A), there is a $D$-path

$$
d=d_{0}, d_{1}, \ldots, d_{m}=a
$$

in $G(T)$.

1.1. If $|w| \geq 3$, there is a path of odd length from $d$ to $d$ in $G(L)$, for example $d, a, b, d$, where $a b d$ is an arbitrary factor of $c(w)$. Therefore, by (B), there is a path of odd length from $d$ to $d$ also in $G(T)$. By Lemma $6, d \in P_{t}$ for some $t$, and thus also $a \in P_{t}$, by Lemma 7 .

We have shown that if $|w| \geq 3$ then $\operatorname{alph}(w) \subseteq P_{t}$ and $g(w)=h(w)$.

2.1. Consider now a word $w$ with $|w|=2$, say $w=a d$.

If $d \in P_{t}$ for some $t$ then the path $d, d_{1}, \ldots, d_{m-1}, a$ guarantees that $a \in P_{t}$ too, by Lemma 7.

Suppose $d \in S$. If $m$ is odd, then by Lemma $5(1), a \sim d$ and we are through. If $m$ is even, then $G(L)$ contains the path

$$
d, d_{1}, \ldots, d_{m-1}, a, d
$$

of odd length. By (B) and Lemma $6, d \in P$, a contradiction. This completes the "only if" part of the proof.

2. Let's approach the question, whether the criterion is necessary.

Clearly, $T$ has to contain a basis of $L$, otherwise it is easy to define periodic morphisms, which agree on $T$, but do not agree lengthwise on $L$.

2.1. Let now $T$ be a subset of $L$, and $D$ a difference support, for which $T$ does not satisfy the condition (A). This means that there are letters $a$ and $b$ which are $D$-connected in $G(L)$, but not in $G(T)$. We define morphisms $g$ and $h$, which agree on $c(T)$, and do not agree on $c(L)$ in the following way. Let

$$
g(x)=a^{i_{x}} \quad h(x)=a^{j_{x}}
$$

for each $x \in X$, which is $D$-connected with $a$ in $G(T)$. Similarly let

$$
g(y)=b^{k_{y}} \quad h(y)=b^{\ell_{y}}
$$

for each $y \in X$, which is not $D$-connected with $a$ in $G(T)$. Then $X=P_{a} \cup P_{b}$. Integers $i_{x}, j_{x}, k_{y}$ and $\ell_{y}$ are such that $D=D(g, h)$.

Let

$$
a=x_{0}, x_{1}, \ldots, x_{k}=b
$$

be a $D$-path in $G(L)$. Since $a \in P_{a}$ and $b \in P_{b}$, there is some $0 \leq i \leq k-1$, such that $x_{i} \in P_{a}$ and $x_{i+1} \in P_{b}$. Moreover, at least one of $x_{i}, x_{i+1}$ is in the difference support. Since there is and edge between $x_{i}$ and $x_{i+1}$ in $G(L)$, there is a word $w \in L$, such that $x_{i}, x_{i+1} \in \operatorname{alph}(w)$. It is manifest that $g$ and $h$ do not agree on $c(w)$.

It remains to prove in this part that $g$ and $h$ agree on $c(T)$. Suppose, on the contrary, that $g$ and $h$ do not agree on $c(u)$ for some $u \in T$. Then, obviously, there are letters $d, e \in \operatorname{alph}(u)$ such that $d \in P_{a}, e \in P_{b}$, and at least one of the letters $e$ and $d$ is an element of $D$. By definition of $g$ and $h$, there is a $D$-path between $a$ and $d$. Then the same path extended by the edge $(d, e)$ is a $D$-path between $a$ and $e$, a contradiction with $e \in P_{b}$. Therefore (A) is a necessary condition. 
2.2. To show that also (B) is necessary, suppose that for some $D \subseteq X$ and $T \subseteq L$ there is a path of odd length from $a \in D$ to itself in $G(L)$, but not in $G(T)$.

Define now morphisms $g$ and $h$ in the following way. Let

$$
g(x)=a b \quad h(x)=a
$$

for each $x \in X$, for which there is a $D$-path from $a$ to $x$ of even length in $G(T)$ (in particular this definition applies $a$ itself); let

$$
g(y)=a \quad h(y)=b a
$$

for each $y \in X$, for which there is a $D$-path from $a$ to $y$ of odd length in $G(T)$; and finally let

$$
g(z)=c^{i_{z}} \quad h(z)=c^{j_{z}}
$$

for each $z \in X$, which is not $D$-connected to $a$ in $G(T)$. The integers $i_{z}$ and $j_{z}$ are chosen to match $D$.

First we have to assure that the definition is correct, which amounts to two claims:

(a) for each $x$ any two $D$-paths in $G(T)$ from $a$ to $x$ have length of the same parity;

(b) each letter connected to $a$ is in $D$.

By assumption, any path $a, \ldots, x, \ldots a$, which links some two $D$-paths from $a$ to $x$ has even length, which proves (a).

Denote by $Y$ all letters connected in $G(T)$ to $a$, including $a$ itself. To prove (b) we claim that each word in $L$, which contains a letter from $Y$ has length 2. Suppose the contrary, and let $w$ contain a factor $c d e$, where $c, d, e$ are (not necessarily distinct) letters from $Y$. (Clearly, if one letter in $\operatorname{alph}(w)$ is in $Y$ then all are.) Let $a, z_{1}, \ldots, z_{\ell}, c$ be a path in $G(T)$. Then

$$
a, z_{1}, \ldots, z_{\ell}, c, d, e, c, z_{\ell}, \ldots, a
$$

has odd length, a contradiction. It is now straightforward to deduce (b) from $a \in D$, and to verify that $g$ and $h$ agree on $c(T)$. On the other hand, Lemma 6 implies that $g$ and $h$ do not agree on $c(L)$.

Let as briefly discuss Theorem 3, which claims, that the decision process based on the criterion of the previous theorem is effective. We suppose that we have given graphs $G(T)$ and $G(L)$. Since the set $X$ of letters is finite, this is a reasonable assumption. The same holds for the basis, which has cardinality at most $|X|$.

We assume that $g(x) h(x)$ is nonempty for each $x \in X$. That's because if $g(x) h(x)$ is empty, the letter $x$ can be omitted from $X$.

The most complicated part of the decision may seem to be the fact that the property is tested over all possible difference supports. There are however also only finitely many of them, and they can be determined by means of basic linear algebra.

\section{EXAMPLES}

In this section we list several important examples which help to illustrate what is going on in search for a Parikh test set.

The first example comes from [Hakala, Kortelainen].

Example 1. Consider alphabet

$$
X_{1}=\left\{a_{1}, \ldots, a_{n}, b_{1}, \ldots, b_{n}, c_{1}, \ldots, c_{n}\right\}
$$


with cardinality $3 n$ and language

$$
L_{1}=c\left(\left\{a_{i} c_{i} b_{j} \mid i, j=1, \ldots, n\right\}\right),
$$

with cardinality of the Parikh map $n^{2}$.

The smallest Parikh test set of $L$ has the cardinality of full $n^{2}$. For example, if all words from $c\left(a_{1} b_{1} c_{1}\right)$ are omitted, the remaining $n^{2}-1$ vectors are not a Parikh test as the following morphisms show:

$$
\begin{aligned}
& g\left(a_{1}\right)=a^{2} \quad g\left(b_{1}\right)=b \quad g\left(c_{1}\right)=a \\
& h\left(a_{1}\right)=a \quad h\left(b_{1}\right)=b \quad h\left(c_{1}\right)=a^{2} \\
& \begin{array}{lll}
g\left(a_{i}\right)=a & g\left(b_{i}\right)=a & g\left(c_{i}\right)=a
\end{array} \\
& h\left(a_{i}\right)=a \quad h\left(b_{i}\right)=a \quad h\left(c_{i}\right)=a
\end{aligned}
$$

The situation changes in the following example, which may seem quite similar to the previous one.

Example 2. Consider alphabet

$$
X_{2}=\left\{a_{1}, \ldots, a_{n}, b_{1}, \ldots, b_{n}\right\},
$$

with cardinality $2 n$, and language

$$
L_{2}=c\left(\left\{a_{i} b_{j} \mid i, j=1, \ldots, n\right\}\right),
$$

with the cardinality of the Parikh map $n^{2}$ as above. The following is the Parikh test set of cardinality $2 n-1$ :

$$
T=\left\{a_{n} b_{i} \mid i=1, \ldots, n\right\} \cup\left\{a_{i} b_{n} \mid i=1, \ldots, n\right\} .
$$

The main reason, why language $L_{2}$ has much smaller Parikh test set than $L_{1}$ is a limited number of difference supports.

The last example shows that the size of Parikh test sets and of classical test sets may differ significantly in some cases.

Example 3. Let

$$
X_{3}=\left\{a_{1}, \ldots, a_{n}, b_{1}, \ldots, b_{n}\right\}
$$

be an alphabet with cardinality $2 n$. Consider language

$$
L_{3}=c\left(\left\{a_{1} \ldots a_{n} b_{i} \mid i=1, \ldots, n\right\}\right) .
$$

The language contains only $n$ Parikh vectors, therefore the Parikh test set is linear. On the other hand any classical test set has at least $\frac{n(n-4)}{4}$ elements. This is caused by the fact that the classical test set has to contain many words with the same Parikh vector.

\section{REFERENCES}

[1] Ismo Hakala and Juha Kortelainen. Polynomial size test sets for commutative languages. RAIRO Inform. Théor. Appl., 31(3):291-304, 1997.

[2] Štěpán Holub and Juha Kortelainen. Linear size test sets for certain commutative languages. Theor. Inform. Appl., 35(5):453-475, 2001. 
Charles University, Faculty of Mathematics and Physics, Department of Algebra, Sokolovská 83, 17586 Praha, Czech Republic

E-mail address: holub@karlin.mff.cuni.cz 